\title{
ARTICLE
}

\section{Positive psychology interventions in patients with medical illness: What predicts improvement in psychological state?}

\author{
Emily H. Feig · Brian C. Healy · Christopher M. Celano · Gholam Reza Nikrahan \\ Judith T. Moskowitz · Jeff C. Huffman
}

\begin{abstract}
Positive psychology interventions (PPIs) have been shown to promote wellbeing in individuals with medical illness, although it is still unknown whether certain patient characteristics make participants more likely to benefit from such interventions. The present study tested whether, using individual patient data across five published PPI studies (three single-arm proof-of-concept trials, one non-randomized controlled trial, and one randomized controlled trial) in medically-ill persons, sociodemographic or psychological factors predicted subsequent change in wellbeing. In 208 participants, lower baseline psychological wellbeing and optimism, and higher symptoms of depression and anxiety were associated with greater improvement in psychological symptoms during the PPI. Other factors were unrelated to symptom changes. In a sub-analysis of controlled studies, there were no group differences in the relationship between baseline factors and changes in wellbeing from pre- to post-intervention. Findings suggest that patients with more severe psychiatric and/or medical comorbidity are no less likely to benefit from a PPI compared to those with higher levels of health, even though these programs do not directly target psychological distress. PPIs may be widely applicable to medical patients, with lower psychological wellbeing a potential predictor of increased benefit.
\end{abstract}

Keywords: positive psychology, behavioral intervention, heart disease, diabetes, depression, anxiety

\section{Introduction}

Positive psychology interventions (PPIs) are treatment methods aiming to promote positive feelings, behaviors, and thoughts via structured activities such as expressing gratitude, performing acts of kindness, and using personal strengths (Sin \& Lyubomirsky, 2009). In healthy individuals, these interventions have consistently improved psychological wellbeing and reduced symptoms of depression (Bolier et al., 2013). They differ from traditional psychological treatments that were developed to address a specific emotional or behavioral problem in that PPIs can benefit individuals with and without mental disorders. The two continua model of mental illness and mental health has demonstrated that mental illness and positive mental health are related, but operate on separate continua; thus the absence of mental illness does not by definition mean one has strong positive 
psychological wellbeing (Keyes, 2005). PPIs operate from this conceptualization; wellbeing is not just the absence of mental disorders, but also the presence of positive psychological resources (Sin \& Lyubomirsky, 2009).

A growing body of literature suggests that PPIs may also improve psychological wellbeing in those with medical conditions, although findings have been inconsistent, with significant variability in the type of intervention delivered and in the patient population. A meta-analysis of PPIs on psychiatric and medical disorders found significant, small effect sizes of the interventions on depression and wellbeing, with no differences in effect size between medical and psychiatric populations (Chakhssi, Kraiss, Sommers-Spijkerman, \& Bohlmeijer, 2018). In addition to the studies reviewed in the meta-analysis, a positive affect intervention was effective in improving medication adherence in a study of 256 African American participants with hypertension, compared to an education only control (Ogedegbe et al., 2012). Further, a positive affect skills intervention for 159 individuals newly diagnosed with HIV led to higher levels of positive affect and less intrusive and avoidant thoughts compared to an attention-matched control up to 15 months after the intervention, but did not lead to significant differences in positive affect immediately after the intervention (Moskowitz et al., 2017). On the other hand, a six-week phone PPI for non-Hispanic white and nonHispanic African American veteran patients with osteoarthritis was not effective, compared to an attention-matched control on improving self-reported pain and functional difficulty (Hausmann et al., 2018). Psychological wellbeing in medical groups is particularly important, as it has been prospectively and independently associated with reduced mortality in those with physical illness (Lamers, Bolier, Westerhof, Smit, \& Bohlmeijer, 2012), a link that is potentially mediated by participation in beneficial health behaviors such as physical activity (Steptoe, Wright, Kunz-Ebrecht, \& Iliffe, 2006), healthy diet (Giltay, Geleijnse, Zitman, Buijsse, \& Kromhout, 2007; Kelloniemi, Ek, \& Laitinen, 2005), smoking abstinence (Giltay et al., 2007), and medication use (Leedham, Meyerowitz, Muirhead, \& Frist, 1995), and that is independent of depression.

Though it appears that PPIs may enhance wellbeing in populations with medical illness (Chakhssi et al., 2018; Huffman et al., 2016; Moskowitz et al., 2017; Ogedegbe et al., 2012), it is unknown whether factors such as gender, age, race/ethnicity, or psychological distress moderate the effect of PPIs. In a meta-analysis of 51 PPIs in healthy individuals (Sin \& Lyubomirsky, 2009), older participants and those with higher levels of depression showed more benefit. The samples in this meta-analysis were primarily younger individuals; only three of the 51 studies tested PPIs in older adults. Further, the interventions were delivered with the primary aim of improving psychological wellbeing. Medical populations are typically older, and the goals of PPIs in this group tend to focus not only on psychological wellbeing but also on health-related quality of life and health behaviors. It has yet to be tested whether these same patterns of response to PPIs would emerge in a medical population, given the differences in age and life situation. Understanding whether participants in the medical setting with certain characteristics respond better to PP programs would be helpful for understanding the variability in results of PP randomized controlled trials thus far, and for maximizing intervention efficacy, allowing providers to offer them to those most likely to benefit. Such an approach is consistent with the increasing focus on personalized approaches to medicine.

Accordingly, across five PPI studies performed and published by our research team in varied medical conditions and settings, we examined, using individual patient data, whether any demographic, medical, and psychological factors predicted subsequent change in psychological 
outcomes, independent of the study population. We chose to include only this set of studies, due to the relative consistency in intervention content (weekly PPI delivered in person or by phone and with accompanying treatment manual) and outcome measures, and so that we could use individual patient level data to improve power of analyses. Based on prior research in healthy samples (Sin \& Lyubomirsky, 2009), we hypothesized that older individuals, and those with higher levels of psychological distress pre-intervention, would show more improvement during the intervention.

\section{Method}

\subsection{Study selection}

In total, our group has conducted six studies of PPIs in medical populations. One of these studies was excluded from this analysis (Nikrahan et al., 2016) because it did not use psychological outcome measures consistent with the other studies; thus we were unable to combine it with the other studies in the analysis. The remaining five studies are detailed below.

\subsection{Description of studies}

All studies involved instruction to complete weekly PP activities, explained by a study trainer, either in person or via phone, and written materials. Participants completed the activities on their own over the week and wrote about them, with review of what they did at the next session. They did not receive reminders between sessions. While intervention content did vary in some ways between studies, they all included a core focus on teaching skills to boost positive thoughts and feelings. At the beginning, each intervention provided education on the rationale for targeting psychological wellbeing to improve health, customized for the target population of that study. All studies also included content on how to develop new skills into habits that could be sustained beyond the study end. Each study was approved by the appropriate institutional review board, and all participants provided written informed consent.

- Positive Emotions After a Coronary Event (PEACE) II (Huffman et al., 2016): This was a nonrandomized proof-of concept trial comparing an eight-week PP phone-based intervention to a treatment-as-usual control for patients after an acute coronary syndrome. The intervention included eight weekly 30-minute individual phone calls plus completion of PP activities between sessions. PP activities were based in gratitude (e.g., identifying positive events in the past week, expressing gratitude via a gratitude letter), strengths (e.g., remembering a past success, identifying and using a personal strength), and meaning (e.g., planning and engaging in acts of kindness, enjoyable and meaningful activities). Participants were recruited from the inpatient cardiac floors at a U.S. academic medical center and enrolled to participate in the study after discharge. Twenty-three participants received the PPI and completed follow-up data; 25 were later recruited and included in the control cohort.

- PEACE III (Celano, Albanese, et al., 2018): This was a randomized factorial trial comparing eight variations of a phone-based PPI in 128 participants after an acute coronary syndrome. Participants were recruited from the inpatient cardiac floors at a U.S. academic medical center and enrolled to participate in the study after hospital discharge. The intervention included eight weekly 30-minute individual phone calls plus completion of PP activities between sessions. Activities were largely the same as those in PEACE II, with the addition of two more: capitalizing 
on positive events and identifying humor in everyday life. Half received booster sessions over the subsequent eight weeks after completing the eight-week intervention; half received an intervention combining PP with motivational interviewing to increase physical activity; and half were instructed to engage in PP activities daily, while the other half were instructed to do so weekly (although all only spoke with their interventionist once per week). There was no control condition.

- Optimism training intervention (Mohammadi et al., 2018): This study, conducted in Iran, was a randomized controlled trial of 61 participants with chronic coronary heart disease who had completed a cardiac rehabilitation program at an academic medical center. Participants were randomly assigned either to an eight-week in-person group optimism training intervention ( $\mathrm{n}=$ 31 ) or an attention-matched educational control condition $(n=30)$. Each group session was two hours long; 17 positive psychological exercises were taught across the eight sessions. The intervention content had some overlap with other studies (e.g., identifying positive events in the past week), but was overall focused on optimism and used different exercises to elicit this (e.g., positive interpretations of life events, optimism in social relationships, detailing one's best possible self and how to take steps to reach it, and using optimism at different times of day). The control condition provided extended education about cardiovascular illness and modifying risk factors.

- Researching Emotions and Cardiac Health (REACH; Celano, Freedman, Beale, Gomez-Bernal, \& Huffman, 2018): This one-armed pilot trial examined a ten-week phone-based PPI in 11 outpatients with heart failure. The intervention included ten weekly 30-minute individual phone calls plus completion of PP activities between sessions. The PP activities were identical to those in PEACE II, with several additional weeks devoted to integrating skills into daily life. Participants were patients at a U.S. academic medical center and were recruited through primary care providers. The intervention also included a motivational interviewing component targeting health behavior engagement. There was no control condition.

- Positive Psychology for Type 2 Diabetes (PP for T2D; DuBois, Millstein, Celano, Wexler, \& Huffman, 2016): This was a one-armed proof-of-concept trial examining an eight-week phonebased PPI in 15 participants with type 2 diabetes. The intervention included eight weekly 30minute individual phone calls plus completion of PP activities between sessions. The PP activities were identical to those in PEACE II. Participants were inpatients or outpatients at a U.S. academic medical center. There was no control condition.

\subsection{Measures}

Questionnaires were completed at the start and end of each intervention. Therefore, the length of time between baseline and follow-up measures was ten weeks for REACH and eight weeks for the other studies.

Depression and anxiety were measured in all five studies, using the Hospital Anxiety and Depression Scale (HADS; Bjelland, Dahl, Haug, \& Neckelmann, 2002), which was developed to measure anxiety and depression in patients with medical illness by excluding physical/somatic symptom assessment. It includes seven items for depression (HADS-D) and seven for anxiety (HADS-A), with a range of scores from 0-21 for each subscale. Both subscales have shown good internal consistency ( $\mathrm{M} \alpha=0.82$ for HADS-D and 0.83 for HADS-A; Bjelland et al., 2002). 
Dispositional optimism was measured in all five studies using the Life Orientation Test - Revised (LOT-R; range 6-24; Scheier, Carver, \& Bridges, 1994), a ten-item scale including six active and four

filler items. This scale has shown good test-retest reliability and internal consistency (Glaesmer et al., 2012; Scheier et al., 1994).

Positive affect was measured in four studies (Celano, Albanese, et al., 2018; Celano, Freedman, et al., 2018; Huffman et al., 2016; Mohammadi et al., 2018) with the positive affect subscale of the Positive and Negative Affect Schedule (PANAS; range 10-50; Watson, Clark, \& Tellegen, 1988), which asks participants to rate their experience of ten types of positive affect over the past week. This measure has shown good internal consistency $(\alpha=0.86)$.

Health-related quality of life was measured in three studies (Celano, Albanese, et al., 2018; Celano, Freedman, et al., 2018; Huffman et al., 2016) with the Medical Outcomes Study Short Form-12 (SF12; Ware, Kosinski, Turner-Bowker, \& Gandek, 2005), a 12-item questionnaire that includes both mental and physical health components (MCS and PCS) with demonstrated internal consistency $(\alpha$ $>0.70$; Luo et al., 2003).

Demographic and health information, including age, gender, marital status, and medical comorbidity, was collected through chart review at time of study enrollment. Age and gender data were available for all five studies, marital status for $4 / 5$ studies ( $n=248$; Celano, Albanese, et al., 2018; Celano, Freedman, et al., 2018; Huffman et al., 2016; Mohammadi et al., 2018), and medical comorbidity for 3/5 studies $(n=187$; Celano, Albanese, et al., 2018; Celano, Freedman, et al., 2018; Huffman et al., 2016).

\subsection{Data analysis}

The association of predictors with the change in each outcome measure was estimated in two ways. Combining data from all five studies into one dataset, we estimated the difference in the change of each outcome with time based on each baseline predictor in all participants who received the PP intervention, using a random intercept linear mixed effects model with main effects for time, the predictor, the predictor-by-time interaction, and a categorical effect of study. The regression coefficient for the interaction term from this model was the focus of the analysis. In a second analysis including only the two studies that included both PPI and control arms (Huffman et al., 2016; Mohammadi et al., 2018), we estimated the difference in the effect of each predictor on the change in each outcome with time, comparing the PPI participants to the control group participants. In this analysis, we fit a random intercept linear mixed effect model with effects for time, PPI, the predictor, and all two-way and three-way interactions. The three-way interaction from this model estimated whether the predictor was associated with a different impact on the change with time, comparing the PPI and control arm. A study effect was also included in the analysis to account for differences between the two studies.

For all analyses, continuous outcome and predictor variables were standardized by dividing by the standard deviation of the variable. This approach allows the mixed model coefficients to estimate the mean change with time in the standardized outcome for a one standard deviation increase in the predictor, which provides a measure of the effect size that can be compared across analyses. All analyses were completed in the statistical package R (www.r-project.org) with the nlme library. A p-value below 0.05 was considered significant, and all tests were two-tailed. 


\section{Results}

\subsection{Participants}

Across studies, 208 participants received a PPI, and 55 were in a control cohort. Mean age was 60.7 years $(\mathrm{SD}=11.4), 46 \%$ of participants were women, and $68 \%$ of those with information provided (n $=169 / 248$ ) were married. Of participants in the United States (four studies; Celano, Albanese, et al., 2018; Celano, Freedman, et al., 2018; DuBois et al., 2016; Huffman et al., 2016), 78\% were white. Participants had a body mass index in the overweight range on average ( $\mathrm{M}=29.4, \mathrm{SD}=5.8) ; 40 \%$ had diabetes, $67 \%$ had hyperlipidemia, and $77 \%$ had hypertension. Summary statistics for each outcome measure at baseline and follow-up are provided for each study in Table 1. A recruitment flowchart for all studies combined can be found in Figure 1 (below).

Table 1. Descriptive information about each measure for each study at the start and end of the intervention

\begin{tabular}{|c|c|c|c|c|c|c|c|}
\hline \multirow[b]{2}{*}{ Study } & \multirow[b]{2}{*}{ Timepoint } & \multicolumn{6}{|c|}{$N, M(S D)$} \\
\hline & & LOT-R $^{\mathrm{a}}$ & PANAS $^{b}$ & $\mathrm{HADS}^{\mathrm{c}} \mathrm{A}^{\mathrm{c}}$ & HADS-D $^{c}$ & SF12 PCS ${ }^{\mathrm{d}}$ & SF12 MCS \\
\hline \multirow{4}{*}{ PEACE II } & \multirow{2}{*}{ Baseline } & $48,23.69$ & $48,35.15$ & $47,7.19$ & $47,4.94$ & $47,41.29$ & $47,47.49$ \\
\hline & & $(4.62)$ & $(7.87)$ & $(5.39)$ & $(4.05)$ & (11.73) & (11.97) \\
\hline & \multirow{2}{*}{8 Weeks } & $42,24.57$ & $42,37.21$ & $41,5.00$ & $41,3.80$ & $44,41.08$ & $44,49.89$ \\
\hline & & $(5.01)$ & $(7.92)$ & $(4.09)$ & $(3.60)$ & $(11.61)$ & $(10.45)$ \\
\hline \multirow{4}{*}{ PEACE III } & \multirow{2}{*}{ Baseline } & $126,22.94$ & $128,36.38$ & $126,7.02$ & $126,4.84$ & $126,36.81$ & $126,47.33$ \\
\hline & & $(5.82)$ & $(7.54)$ & $(4.60)$ & $(3.78)$ & (11.43) & (12.69) \\
\hline & \multirow{2}{*}{8 Weeks } & $89,24.25$ & $85,38.29$ & $87,5.22$ & $87,3.57$ & $89,40.97$ & $89,52.05$ \\
\hline & & $(6.02)$ & $(7.62)$ & $(4.29)$ & $(3.57)$ & (11.46) & $(9.64)$ \\
\hline \multirow{4}{*}{$\begin{array}{l}\text { Optimism } \\
\text { Training }\end{array}$} & \multirow{2}{*}{ Baseline } & $61,19.00$ & $61,27.75$ & $61,7.02$ & $61,7.34$ & - & - \\
\hline & & $(2.85)$ & $(8.00)$ & $(4.18)$ & $(4.26)$ & - & - \\
\hline & \multirow{2}{*}{8 Weeks } & $58,21.00$ & $58,27.81$ & $58,5.31$ & $58,5.71$ & & \\
\hline & & $(3.41)$ & $(7.58)$ & $(3.60)$ & (3.72) & & - \\
\hline \multirow{4}{*}{ REACH } & \multirow{2}{*}{ Baseline } & $11,27.18$ & $11,38.73$ & $11,4.00$ & $11,1.64$ & $11,46.03$ & $11,54.21$ \\
\hline & & $(2.48)$ & (3.35) & $(2.19)$ & $(1.43)$ & $(12.86)$ & $(6.30)$ \\
\hline & \multirow{2}{*}{10 Weeks } & $8,28.75$ & $9,41.56$ & $9,2.22$ & $9,1.44$ & $9,48.43$ & $9,57.70$ \\
\hline & & $(1.39)$ & $(4.77)$ & $(1.64)$ & $(1.42)$ & $(8.68)$ & (3.89) \\
\hline \multirow{4}{*}{ PP for T2D } & \multirow{2}{*}{ Baseline } & $15,19.00$ & 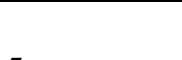 & $15,7.87$ & $15,7.00$ & 2 & 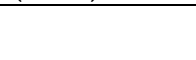 \\
\hline & & $(6.83)$ & - & $(4.19)$ & $(3.66)$ & - & - \\
\hline & \multirow{2}{*}{8 Weeks } & $8,24.38$ & - & $8,6.63$ & $8,3.38$ & - & - \\
\hline & & $(3.34)$ & - & $(3.25)$ & $(1.06)$ & - & - \\
\hline
\end{tabular}

aLife Orientation Test - Revised; bPositive and Negative Affect Scale - positive subscale; ${ }^{c}$ Hospital Anxiety and Depression Scale; dShort Form 12 physical and mental health components 


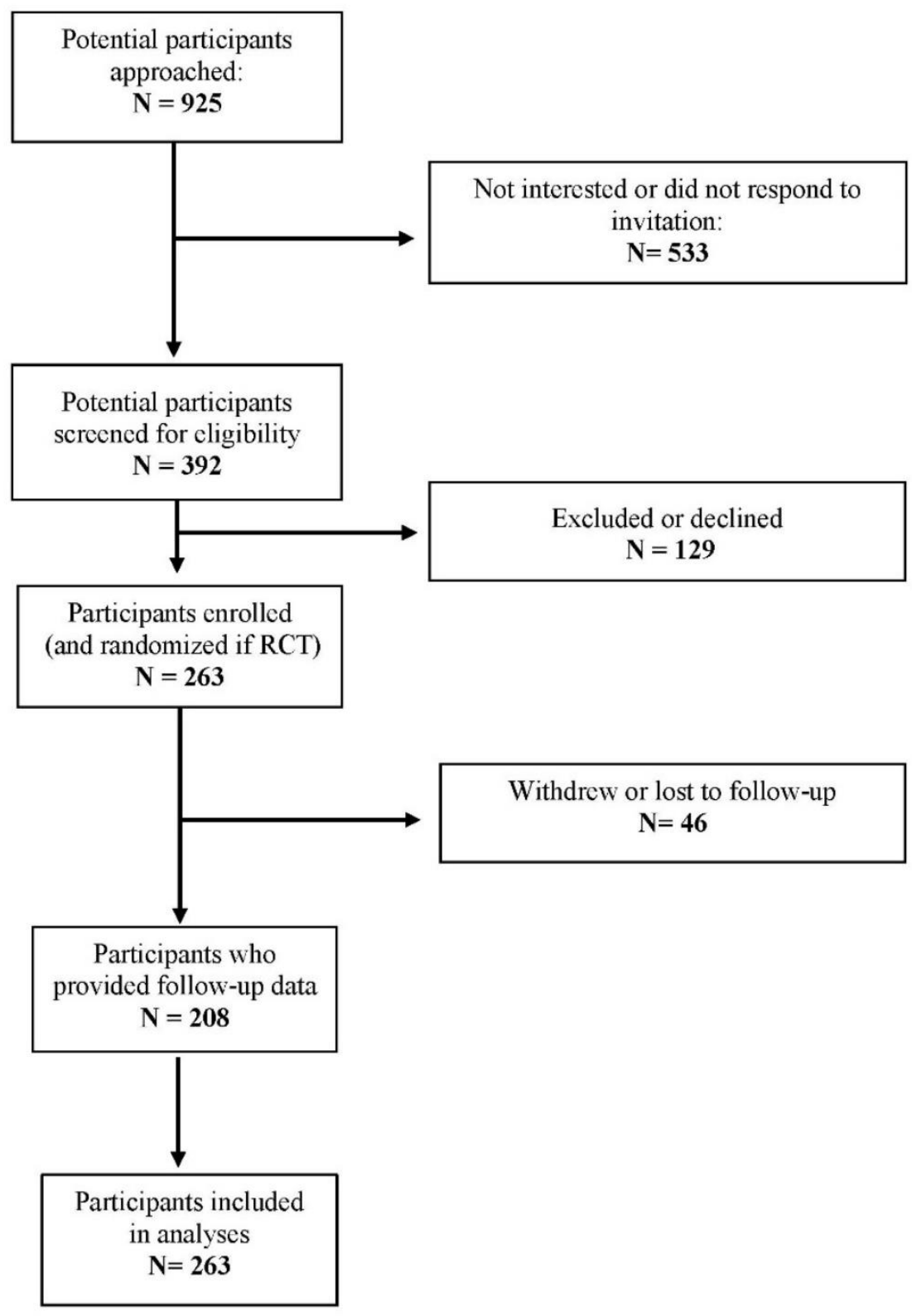

Figure 1. Recruitment flowchart combined across the five studies

\subsection{Predictors of change during the PPI}

Baseline clinical and demographic variables predicted few differences in the change in psychological symptoms with time (Table 2 below). Of all relationships tested, only age was statistically significantly associated with change over time in the depression score, such that younger participants showed a larger pre- to post-intervention decrease in depressive symptoms.

Psychosocial baseline variables predicted changes in outcome over time, such that those with worse baseline functioning had a greater improvement in wellbeing. Specifically, lower baseline mental health-related quality of life was associated with a larger improvement in positive affect, anxiety, and depression (see Table 2 below). Further, lower baseline optimism was associated with 
a larger improvement in depression and anxiety pre- to post-intervention, higher baseline anxiety predicted a larger decrease in depression, and higher baseline depression predicted a larger improvement in anxiety and optimism.

Table 2. Associations between baseline characteristics and change in psychological constructs during the intervention

\begin{tabular}{|c|c|c|c|c|}
\hline \multirow[b]{2}{*}{ Predictor } & \multicolumn{4}{|c|}{ Estimate (95\% Confidence Interval) } \\
\hline & LOT-R $^{\mathrm{b}}$ & PANASc & HADS-A $^{\mathrm{d}}$ & HADS-D $^{\mathrm{d}}$ \\
\hline Age & $-0.08(-0.22,0.07)$ & $-0.07(-0.24,0.10)$ & $0.05(-0.08,0.18)$ & $0.16(0.02,0.30)^{*}$ \\
\hline Gender & $0.14(-0.14,0.43)$ & $-0.04(-0.37,0.30)$ & $0.16(-0.09,0.41)$ & $0.01(-0.27,0.29)$ \\
\hline White & $0.01(-0.41,0.42)$ & $0.22(-0.25,0.69)$ & $-0.16(-0.53,0.21)$ & $0.01(-0.42,0.43)$ \\
\hline $\begin{array}{l}\text { Marital } \\
\text { Status }\end{array}$ & $0.23(-0.08,0.54)$ & $0.00(-0.35,0.35)$ & $0.08(-0.20,0.36)$ & $0.13(-0.18,0.44)$ \\
\hline $\begin{array}{l}\text { Medical } \\
\text { Comorbidity }\end{array}$ & $0.05(-0.12,0.23)$ & $-0.18(-0.38,0.03)^{\wedge}$ & $-0.01(-0.17,0.15)$ & $0.11(-0.06,0.29)$ \\
\hline SF12-PCSa & $0.05(-0.12,0.21)$ & $0.16(-0.03,0.35)^{\wedge}$ & $-0.01(-0.16,0.14)$ & $0.02(-0.14,0.19)$ \\
\hline SF12-MCSa & $-0.07(-0.23,0.09)$ & $-0.26(-0.43,-0.08)^{* *}$ & $0.25(0.11,0.39)^{* *}$ & $0.38(0.23,0.53)^{* * *}$ \\
\hline LOT-R ${ }^{b}$ & -- & $-0.07(-0.24,0.10)$ & $0.26(0.13,0.39)^{* * *}$ & $0.31(0.17,0.45)^{* * *}$ \\
\hline PANASc & $-0.08(-0.22,0.07)$ & -- & $0.16(0.03,0.29)^{*}$ & $0.23(0.09,0.37)^{* *}$ \\
\hline HADS-A ${ }^{d}$ & $0.12(-0.02,0.26)^{\wedge}$ & $0.05(-0.11,0.21)$ & -- & $-0.32(-0.45,-0.19)^{* * *}$ \\
\hline HADS-D ${ }^{d}$ & $0.19(0.05,0.33)^{* *}$ & $0.11(-0.05,0.27)$ & $-0.22(-0.34,-0.10)^{* * *}$ & -- \\
\hline
\end{tabular}

Note: Estimate is the estimated regression coefficient for the interaction term from the linear mixed model, which corresponds to the difference in change with time in standardized outcome for a one standard deviation change in each continuous predictor variable. For dichotomous predictors, the difference in the change with time in standardized outcome between the groups is presented. Analyses included a categorical effect of study. aShort-Form 12 Physical and Mental Health Components. bLife Orientation Test - Revised. cPositive and Negative Affect Schedule - positive affect subscale. ${ }^{d}$ Hospital Anxiety and Depression Scale. ${ }^{\wedge} \mathrm{p}<0.10 ;{ }^{*} \mathrm{p}<$ $0.05 ;{ }^{* *} \mathrm{p}<0.01 ;{ }^{* * *} \mathrm{p}<0.001$

When studies with both a PPI and a control were used to assess for a differential impact of the predictor on the change over time comparing the two arms, there was no significant effect for any of the predictors (Table 3 below).

There were trends that approached significance for a difference between conditions in the relationship between baseline anxiety and change in positive affect, and between baseline depression and change in optimism; those with higher anxiety showed more of an increase in 
positive affect in the PP, compared to the control condition, and those with higher depression showed more of an increase in optimism in the PP, compared to the control condition.

Table 3. Difference in effect of each predictor variable on change in psychological constructs during the intervention in the PPI compared to control condition, for studies that included a control

\begin{tabular}{|c|c|c|c|c|}
\hline & \multicolumn{4}{|c|}{ Estimate (95\% Confidence Interval) } \\
\hline & LOT-R $^{\mathrm{b}}$ & PANASc & HADS-A $^{\mathrm{d}}$ & HADS-D ${ }^{\mathrm{d}}$ \\
\hline Age & $-0.21(-0.68,0.25)$ & $-0.08(-0.50,0.35)$ & $0.18(-0.26,0.62)$ & $0.1(-0.32,0.51)$ \\
\hline Gender & $0.44(-0.55,1.43)$ & $-0.66(-1.56,0.23)$ & $-0.15(-1.08,0.79)$ & $-0.11(-0.97,0.75)$ \\
\hline White & $-0.79(-3.08,1.49)$ & $0.5(-1.40,2.39)$ & $1.18(-1.48,3.84)$ & $1.31(-1.07,3.69)$ \\
\hline Marital Status & $0.99(-0.23,2.21)$ & $-0.49(-1.59,0.61)$ & $0.32(-0.82,1.47)$ & $0.78(-0.27,1.84)$ \\
\hline $\begin{array}{l}\text { Medical } \\
\text { Comorbidity }\end{array}$ & $0.27(-0.50,1.03)$ & $0.00(-0.71,0.70)$ & $0.19(-0.57,0.96)$ & $0.2(-0.56,0.96)$ \\
\hline SF12-PCS ${ }^{a}$ & $0.47(-0.37,1.30)$ & $0.12(-0.57,0.81)$ & $0.09(-0.72,0.90)$ & $0.15(-0.53,0.83)$ \\
\hline SF12-MCSa & $0.18(-0.66,1.02)$ & $-0.35(-0.98,0.28)$ & $-0.37(-1.05,0.31)$ & $-0.04(-0.72,0.63)$ \\
\hline LOT-R ${ }^{b}$ & -- & $0.07(-0.35,0.49)$ & $0.18(-0.23,0.59)$ & $0.11(-0.31,0.54)$ \\
\hline PANASc & $-0.22(-0.66,0.22)$ & -- & $0.25(-0.14,0.64)$ & $0.12(-0.24,0.48)$ \\
\hline HADS-A $^{\mathrm{d}}$ & $0.05(-0.40,0.49)$ & $0.38(-0.01,0.77)^{\wedge}$ & -- & $-0.04(-0.40,0.32)$ \\
\hline HADS-D ${ }^{d}$ & $0.39(-0.07,0.86)^{\wedge}$ & $0.32(-0.06,0.70)$ & $0.06(-0.31,0.42)$ & -- \\
\hline
\end{tabular}

Note: Estimate is the estimated regression coefficient for the three-way interaction term from the linear mixed model, which corresponds to the difference in impact of standardized predictor for continuous predictors or group for dichotomous predictors on change with time in standardized outcome, comparing participants in PP arm to participants in control arm. Results from the two studies with control groups (Huffman et al., 2016; Mohammadi et al., 2018). Analyses included a categorical effect of study. aShort-Form 12 Physical and Mental Health Components. 'bife Orientation Test - Revised. cPositive and Negative Affect Schedule - positive affect subscale. ${ }^{\mathrm{d}}$ Hospital Anxiety and Depression Scale. ${ }^{\wedge} \mathrm{p}<0.10$

\section{Discussion}

In summary, across five PPI studies in several medical populations, baseline clinical and demographic factors were minimally associated with change in psychological wellbeing during the intervention. However, lower baseline psychological wellbeing (measured via mental health-related quality of life, optimism, and positive affect) and higher baseline depression and anxiety were associated with larger improvements in depression and anxiety post-intervention. Additionally, 
lower baseline mental health-related quality of life was associated with a greater increase in positive affect, and higher baseline depression predicted a larger increase in optimism. Of note, in the subanalysis of the two studies with a control condition (Huffman et al., 2016; Mohammadi et al., 2018), the relationship between baseline symptoms and change in depression and anxiety did not differ between groups, though a trend toward greater PP-associated improvements in positive affect and optimism existed among those with higher baseline anxiety and depression, respectively.

The fact that higher baseline distress was associated with greater wellbeing improvement (in the overall analysis) or no less wellbeing improvement (in the sub-analysis) may signal that PPIs can be successfully applied to patients who are experiencing higher psychological distress. It is possible, however, that regression to the mean explains these results, as those with higher baseline symptoms are more likely to reduce symptoms over time, regardless of an intervention. The fact that baseline symptoms did not predict improvement with the PPI compared to the control in the sub-analysis is particularly concerning for this reason, although that sub-analysis is also limited by a smaller sample size. While the present study cannot prove that PPIs work better for those with higher baseline symptoms, it does at least suggest that PPIs are not less effective or harmful for those with depression or anxiety. As PPIs focus on promoting wellbeing rather than directly targeting depression or anxiety, some have wondered whether they could be useful in individuals with significant symptoms of depression or anxiety (Sin \& Lyubomirsky, 2009). Such findings are consistent with prior works in healthy persons that found depression improvements with PPIs to be greatest in those with higher baseline levels of depression (Layous, Chancellor, Lyubomirsky, Wang, \& Doraiswamy, 2011; Sin \& Lyubomirsky, 2009), as well as those finding that high baseline positive affect can limit the effectiveness of PPIs (Froh, Kashdan, Ozimkowski, \& Miller, 2009). On the other hand, Celano and colleagues (2017) found a PPI to be less effective in improving hopelessness than a control condition involving recall of daily events, in a sample of patients recently hospitalized for depression and suicidal ideation. Therefore, PPIs may be best suited for individuals with mild-tomoderate depression. The present study extends these findings to patients experiencing substantial medical illnesses, for whom promotion of wellbeing may be important to recovery and prognosis (Chida \& Steptoe, 2008).

There were no consistent relationships between demographic characteristics and outcomes with these interventions. While younger participants showed a larger decrease in depressive symptoms over time, this is likely explained by their higher baseline depressive symptoms. In their metaanalysis of non-medical populations, Sin and Lyubomirsky (2009) found that PPIs were more effective in older participants, and in their meta-analyses of PP randomized controlled trials, Bolier and colleagues (2013) did not identify any sociodemographic traits that predicted response to the intervention. The lack of a meaningful relationship between demographic variables and change in psychological symptoms in our study suggests that PPIs in persons with medical illness may be applicable to a wide range of individuals, across age, gender, race, and medical comorbidity.

Given that the programs in this analysis all required a trained staff member to deliver the intervention, either in person or over the phone every week, the feasibility and cost of disseminating such a program in a healthcare setting is important to consider. Remote delivery via telephone does improve feasibility in that participants do not have to attend extra appointments, for which transportation can be difficult and costly. However, trained staff must spend significant time on 
intervention delivery for this level of intervention intensity. Adaptation of PPIs to digital platforms (e.g., apps, text messages) may help to reduce cost and interventionist burden in the future.

Strengths of this analysis include a sample of participants from several medical populations who received carefully developed PPIs, and our ability to use all study data to analyze individual participant-level data is a more powerful approach than standard meta-analyses (Panagioti et al., 2016). This was also the first such analysis of predictors of PPI response in patients with medical illness. The analysis also had several limitations. The interventions were all conducted at academic medical centers, and only in patients with heart disease or diabetes, and therefore our findings may not generalize broadly. A lack of a control condition in three of the five studies examined limits our ability to attribute significant relationships to the PPI itself, and some variables (e.g., medical comorbidities) were not measured in all studies. Four studies were quite similar in terms of intervention content, while the fifth differed (Mohammadi et al., 2018), which could obscure trends that would have been seen with a more consistent intervention. On the other hand, PPIs are variable in the literature, and an understanding of predictors of response to different types of PPIs would be useful. Further, multiple analyses increased our risk of type I error. However, this was an exploratory study aimed to identify potential areas for future research, and a Bonferroni correction would cause our significance threshold to be $p=0.0125$, which most of our significance values fell below, suggesting that the findings were largely not due to type I error.

In sum, PPIs appear to be similarly applicable to medically ill patients with a wide range of baseline characteristics, and higher levels of distress do not appear to impede the effect of these programs in medical populations. Future research should examine predictors of response to PPIs in larger, randomized controlled trials to inform delivery of interventions to those most likely to benefit.

\title{
Conflict of interest statement
}

This work was supported by the National Heart, Lung, and Blood Institute grant K23HL123607 (Celano PI) and grant R01HL113272 (to Dr. Huffman), and Tehran Payame Noor University and Isfahan Cardiovascular Research Center (Code: 95112; to Nikrahan PI). The content is solely the responsibility of the authors and does not represent the official views of the National Institutes of Health. The sponsor had no role in the design, analysis, interpretation, or publication of the study. Dr. Celano has received honoraria for talks to Sunovion Pharmaceuticals on topics unrelated to this research. All other authors report no conflicts of interest.

\author{
Authors \\ Emily H. Feig \\ Massachusetts General Hospital \\ Harvard Medical School \\ Brian C. Healy \\ Brigham and Women's Hospital \\ Massachusetts General Hospital \\ Christopher M. Celano \\ Massachusetts General Hospital \\ Harvard Medical School
}


Gholam Reza Nikrahan

Farhangian University

Judith T. Moskowitz

Northwestern University Feinberg School of Medicine

Jeff C. Huffman

Massachusetts General Hospital

Harvard Medical School

jhuffman@mgh.harvard.edu

\section{Publishing Timeline}

Received 21 November 2018

Accepted 5 May 2019

Published 9 July 2019

\section{References}

Bjelland, I., Dahl, A., Haug, T. T., \& Neckelmann, D. (2002). The validity of the Hospital Anxiety and Depression Scale. An updated literature review. Journal of Psychosomatic Research, 52(2), 69-77. https://doi.org/10.1016/S0022-3999(01)00296-3

Bolier, L., Haverman, M., Westerhof, G. J., Riper, H., Smit, F., \& Bohlmeijer, E. (2013). Positive psychology interventions: A meta-analysis of randomized controlled studies. BMC Public Health, 13(1). https://doi.org/10.1186/1471-2458-13-119

Celano, C. M., Albanese, A. M., Millstein, R. A., Mastromauro, C. A., Chung, W.-J., Campbell, K. A., ... Huffman, J. C. (2018). Optimizing a positive psychology intervention to promote health behaviors following an acute coronary syndrome: The Positive Emotions after Acute Coronary Events-III (PEACEIII) randomized factorial trial. Psychosomatic Medicine, 80(6), 526-534. https://doi.org/10.1097/PSY.0000000000000584

Celano, C. M., Beale, E. E., Mastromauro, C. A., Stewart, J. G., Millstein, R. A., Auerbach, R. P., ... Huffman, J. C. (2017). Psychological interventions to reduce suicidality in high-risk patients with major depression: A randomized controlled trial. Psychological Medicine, 47(5), 810-821, https://doi.org/10.1017/S0033291716002798

Celano, C. M., Freedman, M. E., Beale, E. E., Gomez-Bernal, F., \& Huffman, J. C. (2018). A positive psychology intervention to promote health behaviors in heart failure: A proof-of-concept trial: The REACH for Health Study. Journal of Nervous and Mental Disorders, 206(10), 800-808.

Chakhssi, F., Kraiss, J. T., Sommers-Spijkerman, M., \& Bohlmeijer, E. T. (2018). The effect of positive psychology interventions on well-being and distress in clinical samples with psychiatric or somatic disorders: A systematic review and meta-analysis. BMC Psychiatry, 18(1), 211. https://doi.org/10.1186/s12888-018-1739-2

Chida, Y., \& Steptoe, A. (2008). Positive psychological well-being and mortality: A quantitative review of prospective observational studies. Psychosomatic Medicine, 70(7), 741-756. https://doi.org/10.1097/PSY.0b013e31818105ba

DuBois, C. M., Millstein, R. A., Celano, C. M., Wexler, D. J., \& Huffman, J. C. (2016). Feasibility and acceptability of a positive psychological intervention for patients with type 2 diabetes. The Primary Care Companion for CNS Disorders, 18(3). https://doi.org/10.4088/PCC.15m01902 
Froh, J. J., Kashdan, T. B., Ozimkowski, K. M., \& Miller, N. (2009). Who benefits the most from a gratitude intervention in children and adolescents? Examining positive affect as a moderator. Journal of Positive Psychology, 4(5), 408-422. https://doi.org/10.1080/17439760902992464

Giltay, E. J., Geleijnse, J. M., Zitman, F. G., Buijsse, B., \& Kromhout, D. (2007). Lifestyle and dietary correlates of dispositional optimism in men: The Zutphen Elderly Study. Journal of Psychosomatic Research, 63(5), 483-490. https://doi.org/10.1016/j.jpsychores.2007.07.014

Glaesmer, H., Rief, W., Martin, A., Mewes, R., Brähler, E., Zenger, M., \& Hinz, A. (2012). Psychometric properties and population-based norms of the Life Orientation Test Revised (LOT-R). British Journal of Health Psychology, 17(2), 432-445. https://doi.org/10.1111/j.2044-8287.2011.02046.x

Hausmann, L. R. M., Youk, A., Kwoh, C. K,, Gallagher, R. M., Weiner, D. K., Vina, E. R., . . Ibrahim, S. A. (2018). Effect of a positive psychological intervention on pain and functional difficulty among adults with osteoarthritis: A randomized clinical trial + invited commentary + supplemental content. JAMA Network Open, 1(5), 182533. https://doi.org/10.1001/jamanetworkopen.2018.2533

Huffman, J. C., Millstein, R. A., Mastromauro, C. A., Moore, S. V., Celano, C. M., Bedoya, C. A., ... Januzzi, J. L. (2016). A positive psychology intervention for patients with an acute coronary syndrome: Treatment development and proof-of-concept trial. Journal of Happiness Studies, 17(5), 1985-2006. https://doi.org/10.1007/s10902-015-9681-1

Kelloniemi, H., Ek, E., \& Laitinen, J. (2005). Optimism, dietary habits, body mass index and smoking among young Finnish adults. Appetite, 45(2), 169-176. https://doi.org/10.1016/j.appet.2005.05.001

Keyes, C. L. M. (2005). Mental illness and/or mental health? Investigating axioms of the complete state model of health. Journal of Consulting and Clinical Psychology, 73(3), 539-548. https://doi.org/10.1037/0022006X.73.3.539

Lamers, S. M. A., Bolier, L., Westerhof, G. J., Smit, F., \& Bohlmeijer, E. T. (2012). The impact of emotional well-being on long-term recovery and survival in physical illness: A meta-analysis. Journal of Behavioral Medicine, 35(5), 538-547. https://doi.org/10.1007/s10865-011-9379-8

Layous, K., Chancellor, J., Lyubomirsky, S., Wang, L., \& Doraiswamy, P. M. (2011). Delivering happiness: translating positive psychology intervention research for treating major and minor depressive disorders. The Journal of Alternative and Complementary Medicine, 17(8), 675-683. https://doi.org/10.1089/acm.2011.0139

Leedham, B., Meyerowitz, B. E., Muirhead, J., \& Frist, W. H. (1995). Positive expectations predict health after heart transplantation. Health Psychology, 14(1), 74-79. https://doi.org/10.1037/0278-6133.14.1.74

Luo, X., George, M. L., Kakouras, I., Edwards, C. L., Pietrobon, R., Richardson, W., \& Hey, L. (2003). Reliability, validity, and responsiveness of the short form 12-item survey (SF-12) in patients with back pain. Spine, 28(15), 1739-1745. https://doi.org/10.1097/00007632-200308010-00020

Mohammadi, N., Aghayousefi, A., Nikrahan, G. R., Adams, C. N., Alipour, A., Sadeghi, M., .. Huffman, J. C. (2018). A randomized trial of an optimism training intervention in patients with heart disease. General Hospital Psychiatry, 51(October 2017), 46-53. https://doi.org/10.1016/j.genhosppsych.2017.12.004

Moskowitz, J. T., Carrico, A. W., Duncan, L. G., Cohn, M. A., Cheung, E. O., Batchelder, A., ... Folkman, S. (2017). Randomized controlled trial of a positive affect intervention for people newly diagnosed with HIV. Journal of Consulting and Clinical Psychology, 85(5), 409-423. https://doi.org/10.1037/ccp0000188

Nikrahan, G. R., Suarez, L., Asgari, K., Beach, S. R., Celano, C. M., Kalantari, M., . . Huffman, J. C. (2016). Positive psychology interventions for patients with heart disease: A preliminary randomized trial. Psychosomatics, 57(4), 348-358. https://doi.org/10.1016/j.psym.2016.03.003

Ogedegbe, G. O., Boutin-Foster, C., Wells, M. T., Allegrante, J. P., Isen, A. M., Jobe, J. B., \& Charlson, M. E. (2012). A randomized controlled trial of positive-affect intervention and medication adherence in hypertensive African Americans. Archives of Internal Medicine, 172(4), 322-326. https://doi.org/10.1001/archinternmed.2011.1307

Panagioti, M., Bower, P., Kontopantelis, E., Lovell, K., Gilbody, S., Waheed, W., . . Coventry, P. A. (2016). 
Association between chronic physical conditions and the effectiveness of collaborative care for depression: An individual participant data meta-analysis. JAMA Psychiatry, 73(9), 978-989. https://doi.org/10.1001/jamapsychiatry.2016.1794

Scheier, M. F., Carver, C. S., \& Bridges, M. W. (1994). Distinguishing optimism from neuroticism (and trait anxiety, self-mastery, and self-esteem): A reevaluation of the Life Orientation Test. Journal of Personality and Social Psychology, 67(6), 1063-1078. https://doi.org/10.1037/0022-3514.67.6.1063

Sin, N. L., \& Lyubomirsky, S. (2009). Enhancing well-being and alleviating depressive symptoms with positive psychology interventions: A practice-friendly meta-analysis. Journal of Clinical Psychology, 65(5), 467-487. https://doi.org/10.1002/jclp.20593

Steptoe, A., Wright, C., Kunz-Ebrecht, S. R., \& Iliffe, S. (2006). Dispositional optimism and health behaviour in community-dwelling older people: Associations with healthy ageing. British Journal of Health Psychology, 11(1), 71-84. https://doi.org/10.1348/135910705X42850

Ware, J., Kosinski, M., Turner-Bowker, M., \& Gandek, B. (2005). How to Score Version 2 of the SF-12 Health Survey. QualityMetric Incorporated and Health Assessment Lab. Boston, MA: Health Assessment Lab.

Watson, D., Clark, L. A., \& Tellegen, A. (1988). Development and validation of brief measures of positive and negative affect: The PANAS scales. Journal of Personality and Social Psychology, 54(6), 1063-1070. https://doi.org/10.1037/0022-3514.54.6.1063 